\title{
No Relationship Between Preoperative and Early Postoperative Strength After ACL Reconstruction
}

\author{
Pier Paolo Mariani, Luca Laudani, Jacopo E. Rocchi, Arrigo Giombini, and Andrea Macaluso
}

\begin{abstract}
Context: All rehabilitative programs before anterior cruciate ligament (ACL) reconstructive surgery, which are focused on recovery of proprioception and muscular strength, are defined as prehabilitation. While it has shown that prehabilitation positively affects the overall outcome after ACL reconstruction, it is still controversial whether preoperatively enhancing quadriceps strength has some beneficial effect on postoperative strength, mainly during the first period. Objective: To determine whether there is any relationship between preoperative and early postoperative quadriceps strength. Design: Case control. Setting: University research laboratory. Participants: Fifty-nine males (18-33 y; age: 23.69 [0.71] y) who underwent ACL reconstruction with patellar-tendon autograft were examined the day before surgery, and at 60 and 90 days after surgery. Main Outcome Measures: The limb symmetry index (LSI) was quantified for maximal voluntary isometric contraction of the knee extensor muscles and of the knee flexor muscles at $90^{\circ}$ joint angle. A k-means analysis was performed on either quadriceps or hamstrings LSI before surgery to classify the patients in high and low preoperative LSI clusters. Differences in postoperative LSI were then evaluated between the high and low preoperative LSI clusters. Results: Following surgery, there were no differences in the quadriceps LSI between patients with high and low preoperative quadriceps LSI. Sixty days after surgery, the hamstrings LSI was higher in patients with high than low preoperative hamstrings LSI (84.0 [13.0]\% vs $75.4[15.9] \% ; P<.05)$. Conclusions: Findings suggest that quadriceps strength deficit is related to the ACL injury and increases further after the reconstruction without any correlation between the preoperative and postoperative values. Therefore, it appears that there is no need to delay surgery in order to increase the preoperative quadriceps strength before surgery.
\end{abstract}

Keywords: quadriceps strength, prehabilitation, maximal voluntary isometric contraction

Anterior cruciate ligament (ACL) rupture is one of the most common traumatic injuries among physically active individuals. Surgical reconstruction remains the standard approach for athletes who aim to return to high-level sporting activities and aims to reestablish the ligamentous stability of the knee joint. Even if ACL reconstruction (ACLr) has shown good results in terms of knee stability, residual and persistent quadriceps strength deficit is reported as one of the limiting factors in return to preinjury level of function and activity, ${ }^{1-4}$ and this deficit can persist for more than 2 years after surgery. It has been reported that quadriceps weakness is predictive of poor knee function after injury or surgery. ${ }^{5,6}$ Therefore, quadriceps weakness and its potential long-term negative influence on knee function is a major concern after ACL injury and surgery.

It has been reported that preoperatively enhancing quadriceps strength may improve the outcomes of subjects undergoing ACLr, ${ }^{7-9}$ and preoperative rehabilitative programs are commonly believed to be useful to restore strength prior to surgery. ${ }^{10-12}$ All these rehabilitative programs, which are mainly focused on recovery of proprioception and muscular strength, are defined as prehabilitation. The preoperative phase may be useful to reduce the risk of postoperative complications and to improve a successful return to high-level activity. Many studies have confirmed these effects in the long term after surgery with better quadriceps strength

Mariani, Laudani, Giombini, and Macaluso are with the Department of Movement, Human and Health Sciences, University of Rome Foro Italico, Rome, Italy. Laudani is also with the Cardiff School of Sport and Health Sciences, Cardiff Metropolitan University, Cardiff, United Kingdom. Mariani, Rocchi, and Macaluso are with Villa Stuart Sport Clinic, FIFA Medical Centre of Excellence, Rome, Italy. Mariani (ppmariani@virgilio.it and pierpaolo.mariani@uniroma4.it) is corresponding author. and better evaluation scores. ${ }^{10,13,14}$ Therefore, for these reasons, a period of preoperative rehabilitation in order to increase the muscular strength is advised by many surgeons before the surgery. But, in our practice, this period is quite difficult to arrange for athletes who have specific time requirements as to when surgery can be performed. Nevertheless, the relationship between preoperative and postoperative quadriceps strength is more uncertain ${ }^{10}$ when the quadriceps strength is evaluated 3 months after surgery. In addition, most of the previous studies were carried out in cohorts of ACL patients who were not homogeneous, in terms of time elapsed from injury, different graft source, gender, age, and type of muscular assessment.

Therefore, persistent quadriceps weakness following surgery presents a difficult clinical dilemma for the treating clinician. An important underlying factor contributing to this problem is the arthrogenic muscle inhibition, which remains understudied. Different studies ${ }^{15-17}$ have demonstrated that muscular wasting occurs mainly during the first postoperative month, suggesting that there is a surgically induced effect that occurs immediately after ACLr. The purpose of the present study was to determine if there is any relationship between preoperative and early postoperative quadriceps strength after ACLr, mainly during the first 3 months when the greatest wasting of muscular strength occurs. We hypothesized that the early postoperative quadriceps strength was not influenced by its preoperative value but that other factors related to surgery may induce muscular wasting during the first months.

\section{Methods}

We limited our study to the first 3 months for several reasons. The main was that it is that at this time frame, it occurs the most 
significant decline in maximal voluntary isometric contraction (MVIC) and voluntary activation. ${ }^{15,16}$ The mean MVIC decreases of $50 \%$ in comparison with nonaffected limb during the first postoperative month and increases approximately linearly during the following rehabilitation phases. Likewise, Drechsler et $\mathrm{al}^{16}$ reported a side-to-side difference of $59 \%$ at 1 month postoperatively and a decrease to a difference of $34 \% 3$ months after ACLr. Zech et $\mathrm{al}^{15}$ have demonstrated a significant deficit not only for the MVIC but also for voluntary activation during the first postoperative month in the affected limb, suggesting that there is a surgically induced unilateral voluntary activation deficit immediately after ACLr. Another reason to limit our investigation to the early postoperative period was because it is difficult to have a homogenous cohort of patients who follow the same protocol of muscular strengthening with the same intensity and volume of exercises during the late phases of rehabilitation. Differences in age, gender, type of activity, and sports may influence the time committed to rehabilitation with different types and volume of exercises.

\section{Participants}

From January to December 2013, the data of 250 patients who underwent arthroscopic ACLr were serially enrolled as part of a larger series of studies aimed at investigating the effect of ACLr on lower limb health. For this study, 59 patients (18-33 y; age: 23.69 [0.71] y), operated by a single surgeon (P.P.M.) were selected based on the following inclusion criteria: (1) male gender, (2) preinjury Tegner level 9 or 8, (3) occurrence of ACL injury from 30 to 60 days before surgery, (4) isolated ACL injury without meniscal or cartilage lesions as verified at magnetic resonance imaging and at arthroscopy, (5) reconstruction with ipsilateral autologous bone-patellar tendonbone graft, and (6) physical therapy carried out at same center with the same protocol for 3 months. Another reason to limit our investigation to the early postoperative period was because it is difficult to have a homogenous cohort of patients who follow the same protocol of muscular strengthening with the same intensity and volume of exercises during the late phases of rehabilitation. Differences in age, gender, type of activity, and sports may influence the time committed to rehabilitation with different types and volume of exercises.

Exclusion criteria were as follows: (1) history of previous injury or surgery on either knee and (2) presence of swelling or pain during the postoperative phase. None of the patients followed any strengthening rehabilitative protocol prior to surgery. A standardized postoperative rehabilitation protocol was administered at the same center under supervision of physical therapists 5 days per week. Prior to testing, all subjects were informed of all study procedures and provided their informed consent during initial enrolment and prior to data collection. The study also was approved by the University of Rome Foro Italico Review Board.

\section{Data Collection}

Strength testing was carried out in all patients the day before surgery (time 0), and at 60 (time 1) and 90 (time 2) days after surgery. Each patient warmed up on an exercise bicycle for 5 minutes at a low resistance before performing the strength test. All participants were tested for MVIC of the knee extensor muscles at $90^{\circ}$ and of the knee flexor muscles at a $90^{\circ}$ joint angle in both limbs. During the test, participants were seated comfortably on a leg-extension machine (Technogym, Forli-Cesena, Italy) for the knee extension MVIC and on a leg-curl machine (Technogym I) for the knee flexion. Patients were positioned with their trunk erect and fastened by 3 crossing belts on both machines. Muscle force was recorded using a load cell connected to a computerized system unit (MuscleLab 4020e; Bosco-System Technologies, Rieti, Italy). The MVIC task consisted of an increase to a maximum in the force exerted by the leg muscles. Participants were able to follow their performance on the computer screen and were verbally encouraged to achieve a maximum and to maintain it for at least 2 seconds before relaxing. A target line was always set on the computer screen at a value $20 \%$ higher than the best performance. MVIC was calculated as the largest 1-second average reached within any single force recording. For each test $\left(90^{\circ}\right.$ extension MVIC and $90^{\circ}$ flexion MVIC), a minimum of 3 attempts were performed separated by 3 minutes and that with the highest force value was chosen as MVIC. Participants were asked to make a further attempt if the MVIC of their last trial exceeded that of previous trials.

\section{Statistical Analysis}

Side-to-side symmetry was quantified for each isometric MVIC test using the limb symmetry index (LSI), which was calculated as the ratio between the involved and uninvolved limb expressed as a percentage. A 1-way repeated-measures analysis of variance (ANOVA) was used to compare LSI between time 0 , time 1 , and time 2. A k-means cluster analysis was performed on the preoperative LSI to identify any natural grouping that may exist in the involved sample of individuals. The obtained clusters represented individuals characterized by different preoperative LSI, for example, low and high LSI. A 2-factor analysis of variance was then used to evaluate between-clusters differences in LSI at time 1 and time 2. Pearson product-moment correlation coefficients $(r)$ were calculated to evaluate the relationship between LSI at time 0 and at time 1 and time 2 in all patients. The correlation of determination $\left(R^{2}\right)$ between LSI at time 0 and LSI at time 1 and time 2 was also calculated to assess the proportion of the variance of LSI at time 1 and time 2 that is predictable from the LSI at time 0. k-means, ANOVA, and correlation analyses were performed using SPSS software (version 20.0; SPSS, Inc, Chicago, IL). A significance level of $P<.05$ was adopted.

\section{Results}

Descriptive statistics of quadriceps and hamstrings LSI in all patients are reported in Table 1.

\section{Relationship Between Preoperative and Postoperative LSI of Knee Extension MVIC}

Cluster analysis on the LSI of quadriceps MVIC at $90^{\circ}$ knee angle during time 0 led to definition of 2 distinct clusters: One cluster (n: 39) was characterized by high preoperative LSI $(98.8 \pm 10.0 \%)$, whereas the other cluster (n: 20) was characterized by low preoperative LSI $(63.0 \pm 9.4 \%)$. As shown in Figure 1, there were no differences in the LSI between these 2 clusters during both time 1 and time 2 . Accordingly, correlation analysis revealed no significant relationship between preoperative and postoperative LSI of quadriceps.

\section{Relationship Between Preoperative and Postoperative LSI of Knee Flexion MVC}

Cluster analysis on the LSI of hamstrings MVC at $90^{\circ}$ knee angle during time 0 led to definition of 2 distinct clusters: One cluster 
Table 1 Descriptive Statistics for LSI of Quadriceps and Hamstrings Muscles MVC at a $90^{\circ}$ Knee Angle in All Patients Before and After ACLr With Patellar Tendon

\begin{tabular}{lccc}
\hline & Time $\mathbf{0}$ & Time 1 & Time 2 \\
\hline Quadriceps LSI, \% & $83.4 \pm 17.6$ & $50.9 \pm 20.3^{*}$ & $59.6 \pm 18.0^{* * * *}$ \\
Hamstrings LSI, \% & $86.3 \pm 18.2$ & $80.9 \pm 14.6$ & $91.5 \pm 14.6 * *$ \\
\hline
\end{tabular}

Abbreviations: ACLr, anterior cruciate ligament reconstruction; LSI, limb symmetry index; MVC, maximal voluntary contraction; time 0 , preoperative evaluation; time 1, postoperative evaluation 60 days following surgery; time 2, postoperative evaluation 90 days following surgery.

*Significant difference from time 0 . **Significant difference from time 1.

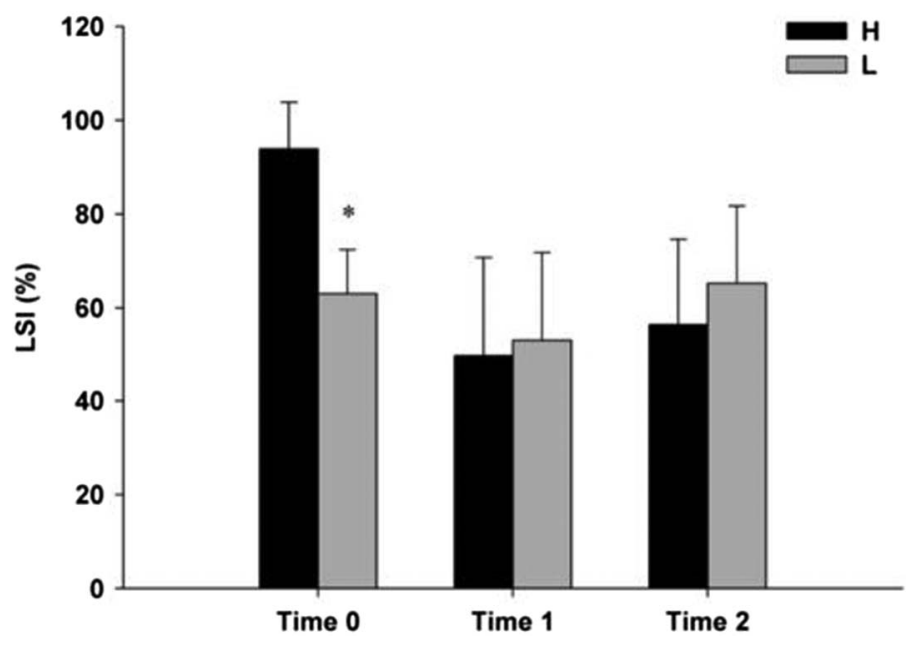

Figure 1 - Limb symmetry indexes during MVC of the quadriceps at a $90^{\circ}$ knee angle in the high (H; n: 39) and low (L; n: 20) LSI clusters of patients at Time 0 (before surgery), Time 1 ( $60 \mathrm{~d}$ after surgery) and Time 2 (90 d after surgery). *Significantly different from H. LSI indicates limb symmetry index; MVC, maximal voluntary contraction.

(n: 38) was characterized by high preoperative LSI $(96.1 \pm 11.8 \%)$, whereas the other cluster (n: 21) was characterized by low preoperative LSI $(68.4 \pm 13.6 \%)$. The statistical analysis of the differences in the LSI of hamstrings between these 2 clusters showed a main effect for the group factor during time $1(F=5.0 ; P<.05)$. As shown in Figure 2, the LSI of hamstrings MVC during time 1 was significantly higher in the high preoperative LSI cluster $(84.0 \pm$ $13.0 \%)$ than in the low preoperative LSI cluster $(75.4 \pm 15.9 \%)$. Consistently, correlation analysis in all patients revealed a significant relationship for the LSI of hamstrings between time 0 and time $1\left(r=.33 ; R^{2}=.10 ; P<.05\right)$.

\section{Discussion}

Our hypothesis was supported as the relationships between preoperative and early postoperative have not been confirmed by our findings. The fast shut down of quadriceps functions, which occurs mainly during the early postoperative period, arises from arthrogenic muscle inhibition, driven by pain, inflammation, and swelling, as well as joint proprioceptors damage. It is regarded as the main mechanism by which quadriceps weakness may persists for a long time after ACL injury or surgery. ${ }^{17-19}$ Clinically, it is important for treating clinicians to devise strategies to overcome this impairment. Many surgeons advise as necessary a period of muscular strengthening before surgery in order to improve outcome after ACLr. For these reasons, during the last years, the concept of "prehabilitation" has emerged to optimize postoperative outcomes of ACLr and to



Figure 2 - Limb symmetry indexes during MVC of the hamstrings at a $90^{\circ}$ knee angle in the high (H; n: 38) and low (L; n: 21) LSI clusters of patients at Time 0 (before surgery), Time 1 (60 days after surgery) and Time 2 (90 days after surgery). *Significantly different from H. LSI indicates limb symmetry index; MVC, maximal voluntary contraction.

reduce the muscular deficits. With the term of prehabilitation, it is defined the period of rehabilitation before surgery, mainly focused on quadriceps strengthening and neuromuscular training. ${ }^{12}$ This preoperative strategy seems suitable for improving the final outcomes assessed by self-report score as IKDC2000 13 or Modified Cincinnati Knee Rating ${ }^{10}$ and to avoid a persistent or prolonged quadriceps strength deficit after surgery. Grindem et al ${ }^{14}$ have described a 5-week progressive prehabilitation program including heavy-resistance strength training and plyometric drills. The patients who followed this program have shown superior knee function both preoperatively and 2 years after surgery. Shaarani et al ${ }^{10}$ provided evidence that the preoperative quadriceps strength predicts a better function of the knee after ACLr regarding the Cincinnati score and the single-legged hop test. Indeed, the prehabilitation increases the patient's compliance during all postoperative phases of rehabilitation and may prepare mentally and physically the patient to follow the long period of rehabilitation. Moreover, when the inflammatory process is particularly acute after injury, the prehabilitation is mandatory to reduce the risk of complications, such as arthrofibrosis.

But the effects of the prehabilitation on the quadriceps strength after surgery are more uncertain. McHugh et $\mathrm{al}^{20}$ have found that preoperative strength of the knee extensors was not a significant predictor of strength following 6 months from surgery. By contrast, Shelbourne and Johnson" have shown that patients with "good" preoperative strength (LSI $>90 \%$ ) had higher postoperative strength than patients with "poor" preoperative strength $(\mathrm{LSI}<75 \%)$. 
Similarly, Eitzen et $\mathrm{al}^{7}$ have shown a positive relationship between preoperative and postoperative strength of the quadriceps at 2 years after ACLr. Lepley et $\mathrm{al}^{21}$ have reported that the quadriceps isometric strength prior to ACLr was positively related to postoperative strength in both the affected and unaffected limbs. Ueda et $\mathrm{al}^{22}$ have shown a positive relation between preoperative and postoperative strength when evaluated at 6 months and 1 year. In all these studies, the assessment of quadriceps strength was performed after 6 months or later from surgery. At shorter time of evaluation, the results are more conflicting. Shaarani et $\mathrm{al}^{10}$ have showed that despite an improvement in terms of outcome, there was a significant decrease in quadriceps peak torque of the injured limb at 12 weeks postoperatively without differences between who were followed a prehabilitation program and the control group. Their results are in accord with our findings.

Therefore, from these studies, it is difficult to draw definitive conclusions and how to improve the quadriceps strength after ACL injury or reconstruction still remains uncertain. Most of the previous studies were carried out in cohorts of ACL patients who were not homogeneous. Moreover, the mean interval from injury to surgery ranged from 3 months to 15 years in the different studies with also differences of gender and of age that may be predictors for delayed muscle recovery. Finally, the method to assess strength also varied greatly. Although isokinetic evaluation is one of the most common methods for strength testing, it should be underlined that the isokinetic test should not be usually performed in the first 2 to 3 months of recovery after ACLr. Recent findings suggest that isometric assessment following ACLr shows construct validity and sensitivity for monitoring recovery of muscle function comparable with those of isokinetic evaluations. ${ }^{23,24}$

Major strengths of the study are therefore: (1) the early assessment of strength, 2 to 3 months after ACL surgery, which rules out other factors underlying strength recovery, such as any change in daily living activities and adherence to the rehabilitation protocol and personal motivations; (2) the homogeneity of patients, as we have included individuals of the same gender, narrow age range, similar activity level with same amount of training who underwent the same surgical technique by a single surgeon at the same center and the same postoperative rehabilitation protocol; and (3) the fact that the patients of the present study were divided into 2 groups (low and high strength) based on the preoperative LSI of either quadriceps or hamstrings MVIC by means of a clustering approach. The adopted k-means cluster analysis is an unsupervised technique, which identifies an optimal data-driven grouping, that is, low and high preoperative LSI clusters, rather than establishing an arbitrary a priori cutoff point of preoperative muscle strength as carried out in previous study. With this approach, we found no differences in postoperative strength of the quadriceps between the low and high preoperative strength clusters, which together with the lack of significant results from the correlation analysis, confirms that there is no relationship between preoperative and postoperative isometric strength of the quadriceps.

There are several weaknesses in our study. First, we have run a prospective observational study in which it has been demonstrated that there is no correlation between preoperative and postoperative strength of the quadriceps in patients who underwent ACLr. Cause-effect relationship is therefore partly speculative. Second, we have limited our observation only to the first 3 months after surgery, and we did not perform any evaluation of our patients until the end of all rehabilitative protocols. Finally, we did not measure arthrogenic muscle inhibition, which is a fundamental factor contributing to the quadriceps muscle weakness in both ACL injured and reconstructed patients.

\section{Conclusions}

The results from the present study indicated that there was no relationship between preoperative and early postoperative isometric strength of the quadriceps following ACLr with patellar tendon. From the clinician's perspective, these results should be considered in the design of preoperative rehabilitation protocols incorporating strengthening exercises. Delaying surgery in order to have a good quadricipes strenght does not seem to be necessary. Our data suggest that quadriceps strength deficit is related to the ACL injury and increases further after the reconstruction without any correlation between the preoperative and postoperative values. After ACLr, early quadriceps exercises are necessary in order to lead to more accelerated muscular recovery.

\section{Acknowledgments}

The authors have no conflicts of interest to disclose. This study was approved by the Review Board of the University of Rome Foro Italico and was, therefore, performed in accordance with the ethical standards laid down in the 1964 Declaration of Helsinki and its later amendments. This work was not funded by any external source.

\section{References}

1. Kobayashi A, Higuchi H, Terauchi M, Kobayashi F, Kimura M, Takagishi K. Muscle performance after anterior cruciate ligament reconstruction. Int Orthop. 2004;28(1):48-51. doi:10.1007/s00264003-0502-5

2. Keays SL, Bullock-Saxton JE, Keays AC, Newcombe PA, Bullock MI. A 6-year follow-up of the effect of graft site on strength, stability, range of motion, function, and joint degeneration after anterior cruciate ligament reconstruction: patellar tendon versus semitendinosus and Gracilis tendon graft. Am J Sports Med. 2007;35(5):729739. PubMed ID: 17322130 doi:10.1177/0363546506298277

3. Lautamies R, Harilainen A, Kettunen J, Sandelin J, Kujala UM. Isokinetic quadriceps and hamstring muscle strength and knee function 5 years after anterior cruciate ligament reconstruction: comparison between bone-patellar tendon-bone and hamstring tendon autografts. Knee Surg Sports Traumatol Arthrosc. 2008;16(11):1009-1016. PubMed ID: 18712355 doi:10.1007/s00167-008-0598-7

4. Risberg MA, Holm I. The long-term effect of 2 postoperative rehabilitation programs after anterior cruciate ligament reconstruction: a randomized controlled clinical trial with 2 years of follow-up. Am J Sports Med. 2009;37(10):1958-1966. PubMed ID: 19556470 doi:10.1177/0363546509335196

5. Lewek M, Rudolph K, Axe M, Snyder-Mackler L. The effect of insufficient quadriceps strength on gait after anterior cruciate ligament reconstruction. Clin Biomech. 2002;17(1):56-63. doi:10.1016/ S0268-0033(01)00097-3

6. Keays SL, Bullock-Saxton JE, Newcombe P, Keays AC. The relationship between knee strength and functional stability before and after anterior cruciate ligament reconstruction. J Orthop Res. 2003;21(2): 231-237. PubMed ID: 12568953 doi:10.1016/S0736-0266(02)00160-2

7. Eitzen I, Holm I, Risberg MA. Preoperative quadriceps strength is a significant predictor of knee function two years after anterior cruciate ligament reconstruction. Br J Sports Med. 2009;43(5):371-376. PubMed ID: 19224907 doi:10.1136/bjsm.2008.057059 
8. de Jong SN, van Caspel DR, van Haeff MJ, Saris DB. Functional assessment and muscle strength before and after reconstruction of chronic anterior cruciate ligament lesions. Arthroscopy. 2007;23(1): 21.e1-21.e11. PubMed ID: 17210423 doi:10.1016/j.arthro.2006. 08.024

9. Shelbourne KD, Johnson BC. Effects of patellar tendon width and preoperative quadriceps strength on strength return after anterior cruciate ligament reconstruction with ipsilateral bone-patellar tendon-bone autograft. Am J Sports Med. 2004;32(6):1474-1478. PubMed ID: 15310573 doi:10.1177/0363546503262171

10. Shaarani SR, O’Hare C, Quinn A, Moyna N, Moran R, O’Byrne JM. Effect of prehabilitation on the outcome of anterior cruciate ligament reconstruction. Am J Sports Med. 2013;41(9):2117-2127. PubMed ID: 23845398 doi:10.1177/0363546513493594

11. Adams D, Logerstedt DS, Hunter-Giordano A, Axe MJ, SnyderMackler L. Current concepts for anterior cruciate ligament reconstruction: a criterion-based rehabilitation progression. J Orthop Sports Phys Ther. 2012;42(7):601-614. PubMed ID: 22402434 doi:10.2519/jospt.2012.3871

12. Wilk KE, Arrigo CA. Rehabilitation principles of the anterior cruciate ligament reconstructed knee: twelve steps for successful progression and return to play. Clin Sports Med. 2017;36(1):189-232. PubMed ID: 27871658 doi:10.1016/j.csm.2016.08.012

13. Logerstedt D, Lynch A, Axe MJ, Snider-Mackler L. Preoperative quadriceps strength predicts IKDC2000 scores 6 months after anterior cruciate ligament reconstruction. Knee. 2013;20(3):208-212. PubMed ID: 23022031 doi:10.1016/j.knee.2012.07.011

14. Grindem H, Granam LP, Risberg MA, Engebresten L, SnyderMackler L, Eitzen I. How does a combined preoperative and postoperative rehabilitation programme influence the outcome of ACL reconstruction 2 years after surgery? A comparison between patients in the Delaware-Oslo ACL Cohort and the Norwegian National Knee Ligament Register. Br J Sports Med. 2015;49(6):385-389. PubMed ID: 25351782 doi:10.1136/bjsports-2014-093891

15. Zech A, Awiszus F, Pfeifer K. Longitudinal changes of neuromuscular quadriceps function after reconstruction of the anterior cruciate ligament. Curr Orthop Pract. 2009;20(3):276-280. doi:10.1097/ BCO.0b013e318193bfc7
16. Drechsler WI, Cramp MC, Scott OM. Changes in muscle strength and EMG median frequency after anterior cruciate ligament reconstruction. Eur J Appl Physiol. 2006;98(6):613-623. PubMed ID: 17036217 doi:10.1007/s00421-006-0311-9

17. Grapar Žargi T, Dobrinič M, Vauhnik J, Kacin A. Factors predicting quadriceps femoris muscle atrophy during the first 12 weeks following anterior cruciate ligament reconstruction. Knee. 2017;24(2): 319-328. doi:10.1016/j.knee.2016.11.003

18. Hart JM, Pietrosimone B, Hertel J, Ingersoll CD. Quadriceps activation following knee injuries: a systematic review. J Athl Train. 2010; 45(1):87-97. doi:10.4085/1062-6050-45.1.87

19. Konishi Y, Fukubayashi T, Takeshita D. Mechanism of quadriceps femoris muscle weakness in patients with anterior cruciate ligament reconstruction. Scand J Med Sci Sports. 2002;12(6):371-375. PubMed ID: 12453165 doi:10.1034/j.1600-0838.2002.01293.x

20. McHugh MP, Tyler TF, Gleim GW, Nicholas SJ. Preoperative indicators of motion loss and weakness following anterior cruciate ligament reconstruction. J Orthop Sports Phys Ther. 1998;27(6):407411. PubMed ID: 9617726 doi:10.2519/jospt.1998.27.6.407

21. Lepley LK, Palmieri-Smith RM. Pre-operative quadriceps activation is related to postoperative activation, not strength, in patients post-ACL reconstruction. Knee Surg Sports Traumatol Arthrosc. 2016;24(1):236-246. PubMed ID: 25315083 doi:10.1007/s00167014-3371-0

22. Ueda Y, Matsushita T, Araki D, et al. Factors affecting quadriceps strength recovery after anterior cruciate ligament reconstruction with hamstring autograft in athletes. Knee Surg Sports Traumatol Arthrosc. 2017;25(10):3213-3219. PubMed ID: 27553297 doi:10. 1007/s00167-016-4296-6

23. Pua YH, Ho JY, Chan SAS, et al. Associations of isokinetic and isotonic knee strength with knee function and activity level after anterior cruciate ligament reconstruction: a prospective cohort study. Knee. 2017;24(5):1067-1074. PubMed ID: 28739425 doi:10.1016/j. knee.2017.06.014

24. Zech A, Witte K, Pfeifer K. Reliability and performance-dependent variations of muscle function variables during isometric knee extension. J Eletromyogr Kinesiol. 2008;18(2):262-269. doi:10.1016/j. jelekin.2006.08.013 